\title{
Magnified endoscopic observation with Fuji Intelligent Chromo Endoscopy of primary duodenal follicular lymphoma
}

Follicular lymphoma occurs rarely in the gastrointestinal tract, comprising only $1 \%-3 \%$ of all B-cell non-Hodgkin lymphomas of the gastrointestinal tract [1]. Prior reports have described cases of follicular lymphoma by using advanced endoscopic imaging [1-4]. With narrowband imaging (NBI) and magnification, follicular lymphoma is reported to show as enhanced whitish areas within enlarged villi and with a coiled vascular pattern $[1,2]$. According to a previous case report [1], the lesion was also identified as whitish small nodules in the jejunum by using Fuji Intelligent Chromo Endoscopy (FICE). However, FICE in combination with magnified endoscopy in follicular lymphoma has not been reported to date. A 63-year-old woman was admitted to our hospital with episodic back and epigastric pain. Upper gastrointestinal endoscopy showed small, whitish polypoid lesions with fold convergence in the second portion of the duodenum ( $\bullet$ Fig. 1 a), and FICE could clearly identify the small polypoid white lesions ( $\bullet$ Fig. 1 b).

Magnified endoscopy showed dilatation and mild irregularity of the villous structures within the lesions ( $\bullet$ Fig. 2 a), and magnifying observation with FICE showed coiled and elongated microvascular patterns within the lesions ( $\bullet$ Fig. $\mathbf{2}$ b).

Histopathology of endoscopic biopsy specimens taken from the lesions showed the follicular structures which consisted of small abnormal lymphoid cells with dense nuclei within the mucosal layer (๑ Fig.3a).

Immunohistochemical analysis revealed that the neoplastic cells were positive for CD10 and CD20 but negative for CD3 and cyclin D. Bcl-2 protein was substantially expressed in the cytoplasm of most of the neoplastic cells ( $\bullet$ Fig. $\mathbf{3 b}$ ), and hence the patient was diagnosed as having duodenal follicular lymphoma.

We report a rare case of duodenal follicular lymphoma observed by magnified endoscopy with FICE, which provides a new type of endoscopic imaging to detect the abnormality in the microvascular and microsurface patterns of this type of gastrointestinal lymphoma.
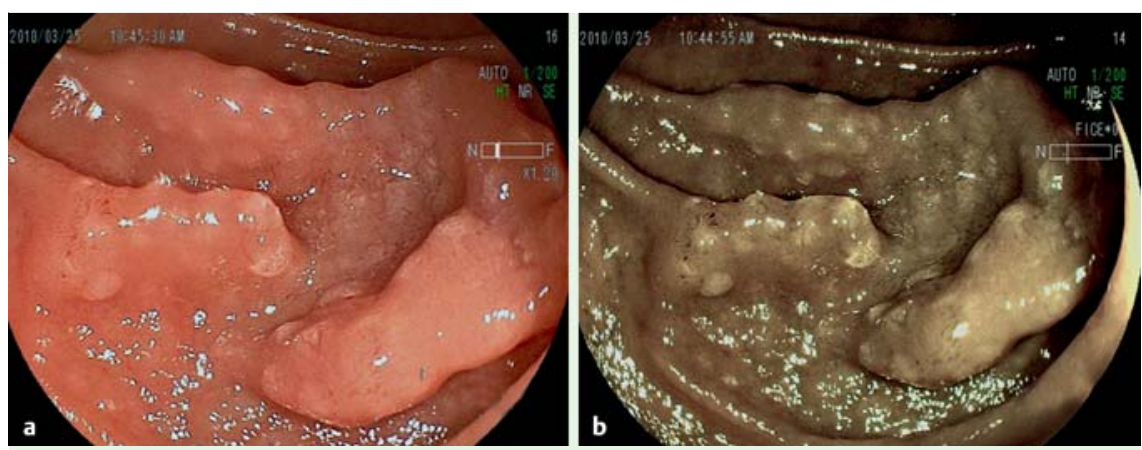

Fig. 1 a Upper gastrointestinal endoscopy shows small, whitish polypoid lesions with fold convergence in the second portion of duodenum. $\mathbf{b}$ Fuji Intelligent Chromo Endoscopy (FICE) clearly identifies the small polypoid white lesions.
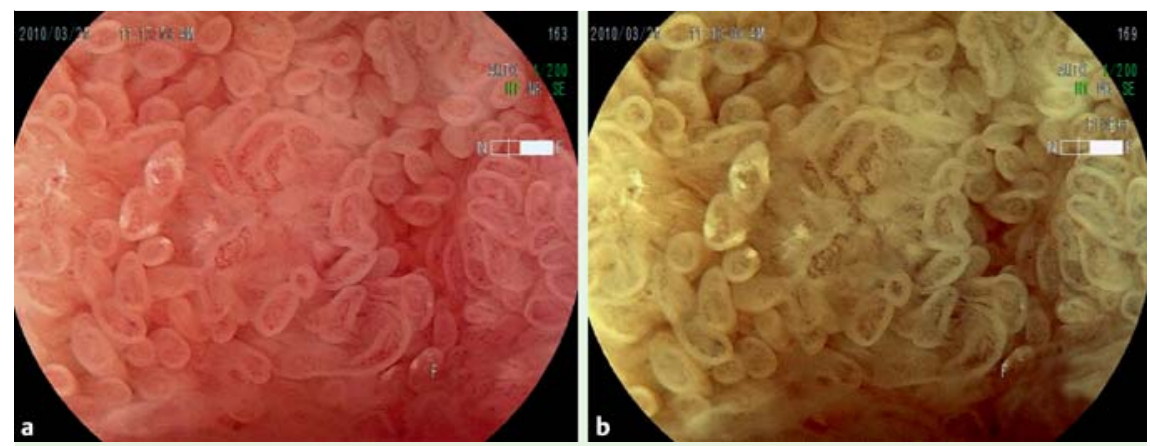

Fig. 2 a Magnified endoscopy shows dilatation and mild irregularity of the villous structures within the lesions. b Fuji Intelligent Chromo Endoscopy (FICE) shows coiled and elongated microvascular patterns within the lesions.
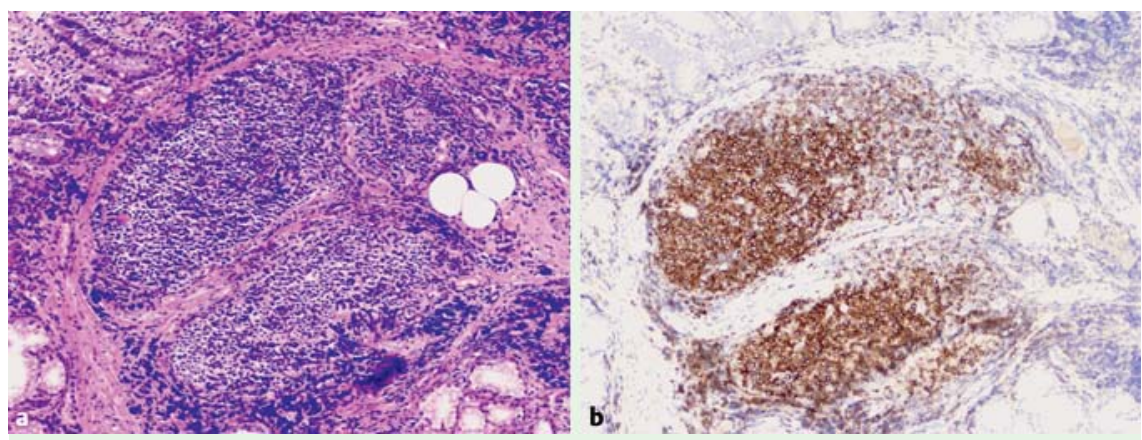

Fig. 3 a Histopathology of endoscopic biopsy specimens taken from the lesions shows the follicular structures consisting of small abnormal lymphoid cells with dense nuclei within the mucosal layer. $\mathbf{b}$ Immunohistochemical analysis shows that $\mathrm{Bcl}-2$ protein was substantially expressed in the cytoplasm of most of the neoplastic cells. 
Competing interests: None

Endoscopy_UCTN_Code_CCL_1AC_2AC

D. Norimura ${ }^{1}$, H. Isomoto ${ }^{1}$, D. Niino ${ }^{2}$,

Y. Akazawa ${ }^{1}$, N. Yamaguchi' ${ }^{1}$, K. Ohnita ${ }^{1}$,

S. Shikuwa ${ }^{1}$, F. Takeshima ${ }^{1}$, K. Nakao ${ }^{1}$

1 Department of Gastroenterology and Hepatology, Nagasaki University Hospital, Nagasaki, Japan

2 Department of Pathology, Kurume University Hospital, Kurume, Japan

\section{References}

1 Chowdhury M, Endo M, Chiba T et al. Characterization of follicular lymphoma in the small intestine using double-balloon endoscopy. Gastroenterol Res Prac 2009; 835258: Epub 2009 Nov 5

2 Inoue N, Isomoto H, Shikuwa S et al. Magnifying endoscopic observation of primary follicular lymphoma of the duodenum by using the narrow-band imaging system. Gastrointest Endosc 2009; 69: 158 - 159

3 Hamada Y, Nimura S, Maeshiro K et al. Follicular lymphoma of the duodenum. Endoscopy $2009 ; 41: 164-165$

4 Higuchi N, Sumida Y, Nakamura K et al. Impact of double-balloon endoscopy on the diagnosis of jejunoileal involvement in primary intestinal follicular lymphomas: a case series. Endoscopy 2009; 41: 175-178
Bibliography

DOI $10.1055 / \mathrm{s}-0030-1255825$

Endoscopy 2010; 42: E320 -E321

(c) Georg Thieme Verlag KG Stuttgart · New York . ISSN 0013-726X

\section{Corresponding author}

D. Norimura, MD

Nagasaki University Hospital

1-7-1 Sakamoto

Nagasaki 852-8501

Japan

Fax: +81-95-819-7482

norir333081@yahoo.co.jp 